\title{
Spectral Variability of Quasars in the Optical Band
}

\author{
Dario Trèvese \\ Dipartimento di Fisica, Università di Roma "La Sapienza", Piazzale A. \\ Moro 2, I-00185 Roma, Italy
}

Fausto Vagnetti

Dipartimento di Fisica, Università di Roma "Tor Vergata", Via della Ricerca Scientifica 1, I-00133 Roma, Italy

\begin{abstract}
We performed a new analysis of the B and $\mathrm{R}$ light curves of a sample of PG QSOs. We confirm a variability-redshift correlation and its interpretation in terms of spectral variability. We find an intra-QSO and an inter-QSO $\alpha-L$ correlation. The former can be explained neither by presence of the host galaxy nor by changes of the accretion rate. Hot spots due to accretion disk instabilities could explain the observations.
\end{abstract}

\section{Introduction}

The physical origin of variability is still substantially unknown. Even restricted to non-blazar objects, the most diverse mechanisms have been considered: gravitational lensing due to intervening matter (Hawkins 1996), supernova explosions (Aretxaga, Cid Fernandes \& Terlevich 1997), instabilities in the accretion disk (Kawaguchi et al. 1998) and star collisions (Torricelli-Ciamponi et al. 2000). A small number of multi-wavelength studies of low redshift objects has provided several indications about different emission components (see Ulrich, Maraschi \& Urry 1997). The most robust and general results come from statistical studies which indicate hardening of the spectrum in the bright phase (Cutri et al. 1985; Edelson, Krolik \& Pike 1990; Kinney et al. 1991; Paltani \& Courvoisier 1994). However, most of the statistical information on AGN variability has been derived, so far, from single-band light curves of magnitude limited samples of objects (Angione et al. 1972; Bonoli et al. 1979; Hawkins 1983; Trèvese et al. 1989; Cristiani, Vio \& Andreani 1990; Trèvese et al. 1994 (T94); Hook et al. 1994; Bershady, Trèvese \& Kron 1998). In this case the strong luminosityredshift (L-z) correlation, caused by the crowding of objects towards the limiting flux makes it difficult to disentangle the intrinsic variability-luminosity ( $v-L$ ) and variability-redshift $(\mathrm{v}-\mathrm{z})$ correlations. Correlation analyses depend on the specific variability index adopted. Giallongo, Trèvese \& Vagnetti (1991) (GTV) found a positive $\mathrm{v}-\mathrm{z}$ correlation, later confirmed by Cristiani et al. (1996), considering a variability index defined on the basis of the rest-frame structure function. The suggestion of GTV that QSOs at high redshift appear more variable since they are observed at a higher rest-frame frequency where the variability is stronger, is consistent with observations. Direct statistical evidence of a spectral 
hardening in the bright phase was found by Giveon et al. (1999) (G99) and by Trèvese, Kron \& Bunone (2001). However, variability mechanisms proposed so far cannot be simply differentiated on qualitative grounds since most of them imply a hardening of the optical-UV spectrum in the bright phase. Here we report on preliminary results of a new analysis (Trèvese \& Vagnetti 2002), based on the data made available to the community by the Wise Observatory group (G99), consisting of $\mathrm{B}$ and $\mathrm{R}$ light curves of 42 nearby, i.e. $z<0.4$, and bright, i.e. $B<16 \mathrm{mag}$ PG QSOs observed with the $1-\mathrm{m}$ Wise Observatory telescope with a median time interval of 39 days for a total duration of 7 years, with r.m.s. photometric uncertainty $\sim 0.01,0.02 \mathrm{mag}$ in B and R, respectively. G99 show a correlation between the color changes $\Delta(B-R)$ and the brightness variations $\Delta B$ and $\Delta R$, corresponding to an average hardening of the spectrum in the bright phase. They do not find a correlation between variability and redshift, at variance with GTV, T94, and Cristiani et al. (1996), but they ascribe this to the difficulty of disentangling $\mathrm{v}-\mathrm{z}, \mathrm{v}-\mathrm{L}$ and $\mathrm{L}-\mathrm{z}$ correlations in the sample, which spans a small redshift interval. We adopt $H_{o}=50 \mathrm{~km} \mathrm{~s}^{-1} \mathrm{Mpc}^{-1}, \mathrm{q}_{\mathrm{o}}=0.5$, unless otherwise stated.

\section{Variability-Redshift Correlation}

To measure the amplitude of variability we define, for each object, the first order structure function, as in Di Clemente et al. (1996) (D96): $S(\tau, \Delta \tau)=$ $\left[\left(\frac{\pi}{2} \mid \overline{m(t+\tau)-m(t)}^{2}-\sigma_{n}^{2}\right)\right]^{1 / 2}$, where $m(t)$ is either the $B$ or the $R$ magnitude, $t$ is the rest-frame time, $\tau$ is the time lag between the observations, $\sigma_{n}$ is the relevant r.m.s. noise and the bar indicates the average taken over all the pairs of observations lying in the time interval $\tau \pm \Delta \tau$. We define four variability indices $S_{i}(\tau \pm \Delta \tau)$, with $i=B, R$, and $\tau=0.3 \pm 0.09 \mathrm{yr}, 2.0 \pm 0.6 \mathrm{yr}$. The subscripts $B$ and $R$ refer to the observing band and the values of $\tau$ and $\Delta \tau$ have been chosen for comparison with previous analyses (D96). None of these four indices shows a significant correlation with redshift when the whole sample is considered, confirming the result of G99. If we restrict the analysis to a magnitude bin $-23.5<M_{B}<-22.5$, around the average absolute magnitude of the sample $\left\langle M_{B}\right\rangle=-22.75$, to disentangle $\mathrm{v}$-L end L-z correlations, we find a $\mathrm{v}-\mathrm{z}$ correlation coefficient $r_{v, z}=0.39$, which is marginally significant $(P(>r)=0.09)$ despite the small number of objects (19) in the bin.

We take the ensemble averages of the four variability indices defined above, over the same subsample of 19 objects, to examine the dependence of variability on redshift. For each observing band we compute the average rest-frame observing frequency of the sample. We must take into account the dependence of variability on magnitude for comparison with D96, reducing $S$ by an amount $\Delta S=\left(\partial S / \partial M_{B}\right) \Delta M_{B}$, where for $S\left(M_{B}\right)$ we adopt model A of Cristiani et al. (1996) and $\Delta M_{B}$ is the difference between the average absolute magnitudes of the present sample and the sample of D96. The increase of variability with the observing frequency is shown in Figure 1a, where the new points appear consistent with the previous results. The general trend can be quantified as: $\partial S_{i} / \partial \log \nu_{r e s t}=\partial S_{i} / \partial \log (1+z) \simeq 0.25-0.3$ in agreement with GTV, Cristiani et al. (1996) and D96. Despite poor statistics, the present results confirm 
the increase of variability with redshift and its interpretation in terms of an average increase of the amplitude of variability with frequency.

\section{Spectral Variability}

Direct evidence of an average hardening of the SED in the bright phase was obtained by Trèvese, Kron, \& Bunone (2001) from $U, B, F, N$ photometry of a sample of 40 QSOs observed at two epochs. A statistical analysis of SED variations of individual objects is made possible by the $B$ and $R$ light curves of G99, containing on average 40 points per object and allowing the computation of the time dependent spectral slope for each QSO:

$$
\alpha(t)=-\left(0.4\left[(B-R)-\left(B_{o}-R_{o}\right)\right]\right) / \log \frac{\lambda_{R}}{\lambda_{B}}-2
$$

where $B_{o}, R_{o}$ are the zero points of $\mathrm{B}, \mathrm{R}$ photometric bands. This is reported in Figure $1 \mathrm{~b}$ as a function of the relevant intrinsic luminosity $L_{\nu_{B}}$. Each small cloud of points represents an individual QSO at different epochs. The relevant regression lines are reported for each cloud. They show, with a few exceptions, a positive correlation between the spectral slope and the intrinsic luminosity, which we call the intra-QSO $\alpha-L$ correlation. The distribution of clouds in the $\alpha-L_{\nu_{B}}$ plane also shows an inter-QSO $\alpha-L$ correlation, indicated by the thick regression lines, corresponding to a highly significant $\left(P(>r)=6 \cdot 10^{-5}\right)$ correlation coefficient. $r_{\alpha-L}=0.58$.

We quantify the spectral variations of individual objects by a spectral variability parameter (SVP) which represents the spectral slope changes per unit log-luminosity change: $\beta(\tau) \equiv \frac{\alpha(t+\tau)-\alpha(t)}{\log L_{B}(t+\tau)-\log L_{B}(t)}$. where $L_{B}(t)$ is the luminosity in the $B$ band and $\tau$ is an appropriate time delay. For the following analysis we define the SVP of each QSO as the mean value $\beta_{m}$ in a single bin $0<\tau<1000 \mathrm{~d}$. In Figure 2, $\beta_{m}$ values for the 42 QSOs are reported versus the relevant time average $\bar{\alpha}$. The dot-dashed line represents a black body of fixed area and varying temperature whose spectral slope is $\alpha_{B B}(x)=3-x e^{x} /\left(e^{x}-1\right)$ and the SVP is $(d \alpha / d T) /\left(d \log B_{\nu} / d T\right)=(\ln 10)\left[1-x /\left(e^{x}-1\right)\right] \equiv \beta_{B B}(x)$, where $x \equiv h \nu / k T$, with $T, h, k$ equal to the temperature, Planck, and Boltzmann constants respectively. Typical values $\langle\bar{\alpha}\rangle=-0.2 \pm 1.0,\left\langle\beta_{m}\right\rangle=2.2 \pm 0.9$, correspond to temperature changes of a black body of $T \approx 10^{4} \mathrm{~K}$, in approximate agreement with the result of Trèvese, Kron \& Bunone (2001).

According to Cid Fernandes et al. (2000), color changes in G99 data imply the presence of a constant spectral component, redder than the variable one, which could be identified with either the non-flaring part of the QSO spectrum or with the host galaxy, also considered by Romano \& Peterson (1998). We evaluate the latter effect through numerical simulations based on templates of the QSO and host galaxy SEDs from Elvis et al. (1994) (see also Trèvese \& Vagnetti (2001)). QSO and host spectra are added with a relative weight $\eta \equiv$ $\log \left(L_{H}^{Q} / L_{H}^{g}\right)$, where $L_{H}^{Q}$ and $L_{H}^{g}$ are the total $H$ band luminosities of the QSO and the host galaxy, respectively. We explore a wider range $-3<\eta<3$. Variability is represented by small changes $\Delta \eta$ deriving from a luminosity change which corresponds to the r.m.s. variability $\sigma_{B}=0.16 \mathrm{mag}$. For each synthetic spectrum, representing the QSO plus host SED at a given time, we compute $\alpha(\bar{\nu}, t) \equiv\left(\partial \log L_{\nu} / \partial \log \nu\right)_{\nu=\bar{\nu}}, \bar{\nu}=\sqrt{\nu_{B} \nu_{R}}$, then we derive the SVP $\beta$. The 

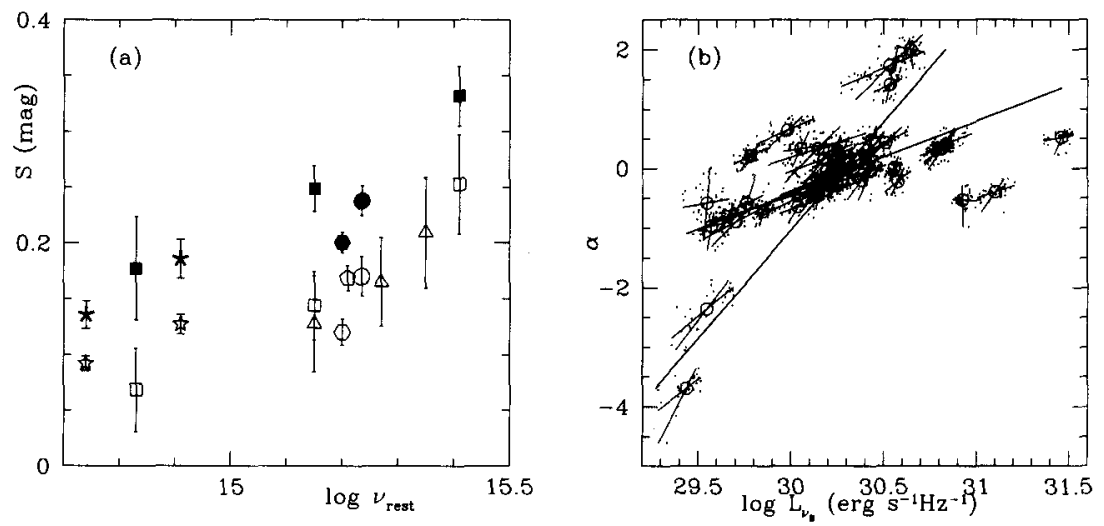

Figure 1. (a) Variability $S$ versus rest-frame frequency for various QSO samples. Filled symbols correspond to $\tau=0.3 \pm 0.09 \mathrm{yr}$ and open symbols correspond to $\tau=2.0 \pm 0.3$ yr. Stars: Trevese \& Vagnetti 2002; squares: D96; triangles: Cimatti et al. 1993; pentagons: Hook et al. 1994; hexagons: Cristiani et al. 1990; circles: T94. (b) Instantaneous spectral slope $\alpha$ versus monochromatic luminosity $L_{\nu_{B}}$. Regression lines $\alpha-L_{\nu_{B}}$ are reported for each QSO (thin lines, intra-QSO correlation), and for the population (thick lines, inter-QSO correlation).

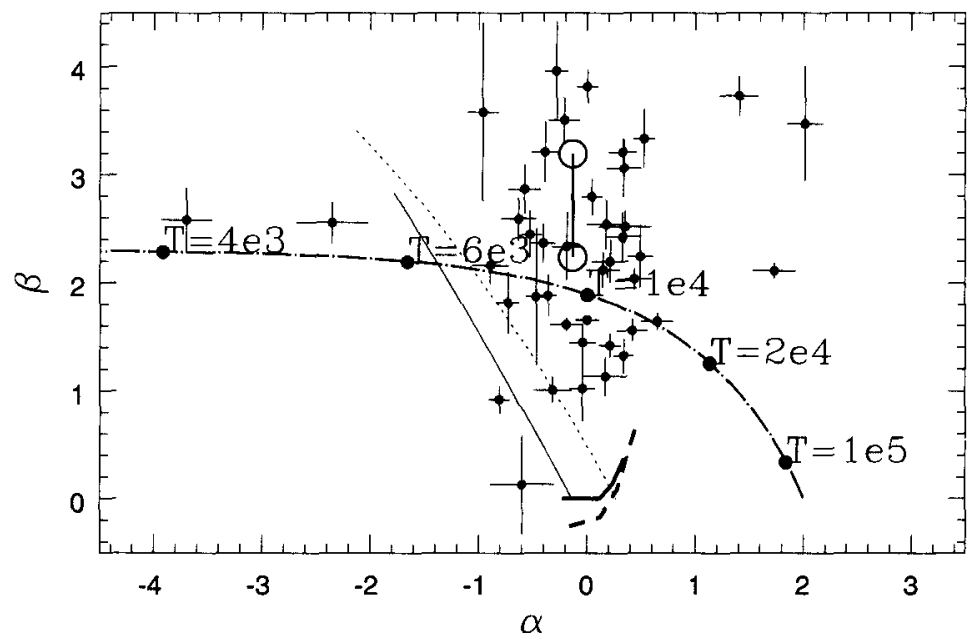

Figure 2. The spectral variability parameter $\beta_{m}$ versus the average spectral slope for each QSO of the sample. Dot-dashed line: black bodies of different $T$; continuous line: the effect of the host galaxy, for $z=0$; dotted line: the same for $z=0.4$; thick continuous line: spectral variability caused by an increase of $\dot{m}$ from 0.1 to 0.3 from the Siemiginowska et al. (1995) model; thick dashed line: the same for $\dot{m}$ increasing from 0.3 to 0.8 . Large open circles: hot spot model for $T=2 \cdot 10^{5} \mathrm{~K}$ (upper) and $T=2 \cdot 10^{4} \mathrm{~K}$ (lower). 
result is shown in Figure 2. The same computation is repeated for the maximum redshift of the G99 sample, $z=0.4$, to check the dependence of the result on QSO redshift. Although an appropriate choice of $\eta$ can reproduce the observed $\beta$ or $\alpha$ separately, the curves are clearly shifted with respect to the distribution of the observational points, indicating that the host galaxy is not sufficient to account for the observed changes of the spectral shape. This also implies that spectral variations are intrinsic to the active nucleus and that the constant red continuum, resulting from the analysis of Cid Fernandes et al. (2000), cannot be identified with the host galaxy but it must be, at least in part, due to the nucleus itself. Thus it could be identified with the spectrum of the non-flaring part of the accretion disk.

We can check whether a change of the accretion rate $\dot{M}$ can account for the observed variations of the spectral shape. We considered the accretion disk model of Siemiginowska et al. (1995) (S95), corresponding to a Kerr metric and modified black body SED. A grid of models has been considered for $\log M / M_{\odot}=7.0,8.0,9.0,10.0, \dot{m} \equiv \dot{M} c^{2} / L_{E}=0.1,0.3,0.8$ (where $L_{E}$ is the Eddington luminosity $L_{E}=4 \pi G c m_{p} / \sigma_{e} M$ with the usual meaning of symbols) and inclination $\mu \equiv \cos \theta=1,0.75,0.5,0.25,0.1$. A change of $\dot{M}$ produces a variation of both luminosity and the SED shape. It is interesting to see how the spectral changes between two $\dot{M}$ states compare with the observed ones, as done by S95 and Tripp et al. (1994). The two curves on the bottom right of Figure 2 represent $\beta$ and $\alpha$ changes obtained by varying $M$ (from right to left), as computed for two different values of $\dot{m}$ and for $\mu=1$, from the Kerr metric, modified blackbody model of S95 (their Table 4). The spectral variations are clearly smaller, on average, than the observed ones, meaning that a transition, e.g., from a lower to a higher $\dot{M}$ regime implies a larger luminosity change for a given slope variation.

This result suggests that transient phenomena, like hot spots produced on the accretion disk by instability (Kawaguchi et al. 1998), instead of a transition to a new equilibrium state, may better explain the relatively large changes of the local spectral slope. The available models of instability phenomena do not provide a spectrum of the hot spot. Thus we try a simple "model" based on the addition of a black body flare to the disk SED, represented by the average QSO SED of Elvis et al. (1994). $\Delta B=0.16 \mathrm{mag}$, which corresponds to the r.m.s. variability of the sample, can be obtained by a hot spot of $T_{B B} \approx 2 \cdot 10^{5} \mathrm{~K}$ and $A=5 \cdot 10^{30} \mathrm{~cm}^{2}$, producing $\beta=3.2$, or $T_{B B} \approx 2 \cdot 10^{4} \mathrm{~K}, A=1,3 \cdot 10^{32} \mathrm{~cm}^{2}$, giving $\beta=2.2$, as shown by the large circles in Figure 2. A sudden heating of a fraction of the disk surface is thus capable of producing the observed change of the SED in the $B$ and $R$ bands and the relevant intra-QSO $\alpha$-L correlation.

\section{References}

Angione, R. J., \& Smith, H. J. 1972, IAU Symp. 44, 171

Aretxaga, I., Cid Fernades, \& Terlevich, R., 1997, MNRAS, 286, 271

Bershady, M. A., Trèvese, D., \& Kron, R. G. 1998, ApJ, 496, 103

Bonoli, F., Braccesi, A., Federici, L., \& Zitelli, V., 1979, A\&AS, 35, 391

Cid Fernandes, R., Sodrè, L., Vieira da Silva, L., 2000 ApJ, 544, 123 
Cimatti, A., Zamorani, G., \& Marano, B. 1993, MNRAS, 263, 236

Cristiani, S., Vio, R., \& Andreani, P. 1990, AJ, 100, 56

Cristiani, S., et al 1996, A\&A, 306, 395

Cutri, R. M., et al. 1985, ApJ, 296, 423

Di Clemente, A., et al. 1996, ApJ, 463, 466

Edelson, R.A., Krolik, J. H.,\& Pike, G. F. 1990, ApJ, 359, 86

Elvis, M., et al. 1994, ApJS, 95, 1

Giallongo E., Trèvese D., Vagnetti F.: 1991, ApJ, 377, 345

Giveon, U., et al. 1999, MNRAS, 306, 637

Hawkins, M. R. S., 1983, MNRAS, 202, 571

Hawkins, M. R. S., 1996, MNRAS, 278, 787

Hook, I. M., et al. 1994, MNRAS, 268, 305

Kawaguchi, T., et al. 1998, ApJ, 504, 671

Kinney, A. L., et al. 1991, ApJS, 75, 645

Paltani, S., \& Courvoisier, T. J.-L. 1994, A\&A, 291, 74

Romano, P., \& Peterson, B. M., 1998, ASP Conf. Ser. 175, 55

Siemiginowska, et al. 1995, ApJ, 454, 77

Torricelli-Ciamponi, G., et al. 2000, A\&A, 358, 57

Trèvese, D., Kron, R. G., \& Bunone A., 2001, ApJ, 551, 103

Trèvese, D., et al. 1994, ApJ, 433, 494 (T94)

Trèvese, D., et al. 1989, AJ, 98, 108

Trèvese, D., \& Vagnetti, F., 2001, in 'QSO Hosts and their Environments', I. Marquez et al. Eds., in press (astro-ph/0102252)

Trèvese, D., \& Vagnetti, F., 2002, ApJ, (in press) (astro-ph/0110075)

Tripp, T. M., Bechtold, J., \& Green, R. F. 1994, ApJ, 433, 533

Ulrich, M. H., Maraschi L., \& Urry C. M. 1997, ARA\&A, 35, 445 